\title{
A Numerical Study of Si-TMD Contact with n/p Type Operation and Interface Barrier Reduction for Sub-5 nm Monolayer MoS2 FET
}

\author{
Ying-Tsan Tang ${ }^{1}$, Kai-Shin Li ${ }^{1}$, Lain-Jong Li ${ }^{2}$, Ming-Yang Li ${ }^{2}, 3$, Chang-Hsien Lin ${ }^{1}$, Yi-Ju Chen ${ }^{1}$, Chun-Chi Chen ${ }^{1}$, \\ Chuan-Jung Su${ }^{1}$, Bo-Wei $\mathrm{Wu}^{1}$, Cheng-San Wu${ }^{1}$, Min-Cheng Chen ${ }^{1}$, Jia-Min Shieh ${ }^{1}$, Wen-Kuan Yeh ${ }^{1}$, Po-Cheng Su${ }^{3}$, \\ Tahui Wang ${ }^{3}$, Fu-Liang Yang ${ }^{4}$, Chenming $\mathrm{Hu}^{5}$ \\ ${ }^{1}$ National Nano Device Laboratories, National Applied Research Laboratories, Taiwan; \\ ${ }^{2}$ Physical Sci. and Eng., King Abdullah University of Sci. and Technology, Kingdom of Saudi Arabia; \\ ${ }^{3}$ Dept. of Electronics Eng., National Chiao-Tung University, Taiwan; \\ ${ }^{4}$ Research Center for Applied Sci., Academia Sinica, Taiwan; \\ ${ }^{5}$ Dept. of Electrical Eng. and Computer Science, University of California, Berkeley, USA; \\ Tel: (886) 357-26100 ext. 7511,7706, Fax: (886) 357-26109, Email: ksli@narlabs.org.tw; mcchen@narlabs.org.tw
}

\begin{abstract}
An atomic-scale numerical study of $\mathrm{Si}$ contact with transition metal dichalcogenides (TMD) semiconductor materials is proposed by first-principles simulation for the first time. The monolayer $\mathrm{MoS}_{2}$ channel can be operated as both of n- and p-type FET by properly doping Si S/D to adjust the TMD channel potential. The gradient $\mathrm{MoS}_{\mathrm{x}}$ junction of dichalcogenide vacancies enables $\mathrm{Si}-\mathrm{MoS}_{2}$ contact resistance lower than $100 \Omega-\mu \mathrm{m}$ for interface Schottky barrier height reduction. The compact Si-MoS interface study can potentially provide monolayer TMD contact design guideline for the sub-5 nm TMD FET fabrication technology.
\end{abstract}

\section{Introduction}

Traditional Si channel field effect transistors (FET) have faced physical limitation in nano-scale transistor, there are many alternative novel materials were proposed to meet the channel scaling of Moore's law [1]. First monolayer $\mathrm{MoS}_{2}$-FET was proposed in 2011 [2] since advanced 2D semiconductor material is an ideal channel material for its unique sub-nm monolayer ultra-thin body [3] and good transport property at extremely small thinness [4]. Moreover, there are many lately published results [5-9] demonstrated TMD-FET have great potential in sub-10nm channel length FET application (Table 1). However, TMD contacts heterogeneous integration still is a big challenge in $\mathrm{n}$ - and $\mathrm{p}$ channel formation and contact resistance reduction. In this paper, the numerical study of TMD contacts between monolayer $\mathrm{MoS}_{2}$ and bulk Si with unique contact techniques was presented in atomic scale first-principles simulations. The design of TMD FET structure for CMOS compatible process is still lack. From this work, we also show that the $\mathrm{n} / \mathrm{p}$ FET operation very well at the same TMD channel layer with properly $\mathrm{n} / \mathrm{p}$ type doping at $\mathrm{Si}$ S/D contact for sub-5nm channel $\mathrm{MoS}_{2}$ FET, which could be compared with our experimental result[7]. We can also predict a minimum achievable contact resistance well below $100 \Omega-\mu \mathrm{m}$ with dichalcogenide vacancies in the gradient $\mathrm{Si}-\mathrm{MoS}_{\mathrm{x}}$ junction.

\section{Simulation Approach}

This numerical DFT calculations made use of Atomistix ToolKit (ATK2015). The whole process is designed by Fig1. Firstly, one may build the interfacial structure of $\mathrm{MoS}_{2}-\mathrm{Si}$ with assistance of minimum strain package in ATK, which greatly shorten calculation time of the unit cell geometry optimization. To sample the K-space of contact region, $9 \mathrm{x} 13 \times 1 \mathrm{k}$ points were adopted, higher $\mathrm{k}$ points is required for repetition bulk calculation. The density mesh grid cut-off follows previous study [9] with 200Ry and an acceptable geometry optimize force as $0.05 \mathrm{eV} / \hat{A}$. The HartwigsenGoedecker-Hutter (HGH-T8) pseudo-potential basis is chosen for closely matching experimental value of band gap $1.8 \mathrm{eV}$ in $\mathrm{MoS}_{2}$. To describe many particle internal interaction and van der Waals interactions between layers, an exchange and correlation interactions of the generalized gradient approximation (GGA) and Grimme DFT-D3 are used here. Substituting all above parameters into Kohn-Shan Equation leads to a self-consisted calculation of electron density. Once the charge density is converged with minimum energy, the bulk simulation is completed, and associated physical quantities of electron density, band structure and density of state (DOS) is found. Current simulation is associated with electron transport calculation. Generally the system consisted of a central region (channel- $\mathrm{MoS}_{2}$ ) and two contact (scattering in $\mathrm{MoS}_{2}-\mathrm{Si}$ ) region. Such canonical system calculation can be expanded with previously optimized bulk structure's state and using non-equilibrium Green's function (NEGF) method to calculate transmission spectrum. Once the zero-bias transmission spectrum is calculated, one may speed up finite-bias ones by initializing a new calculation from previous converged state of another. Integrating the transmission coefficient over the bias windows gives the transport current. Hence a useful $\mathrm{MoS}_{2}$ FET based on first-principles current simulation is archived.

Many experimental literatures [1-8] demonstrated that solid CMOS processes on $\mathrm{Si}$ substrate can be very compatibility for sub-10 nm channel length $\mathrm{MoS}_{2}$ FET manufacturing. Therefore, Fig. 2 (a) shows the simulated schematic of a monolayer $\mathrm{MoS}_{2}$ FET device with $\mathrm{Si}$ source/drain along the channel in this work. Furthermore, to 
reduce the Schottky barrier and improve Si-MoS 2 contacts, we need understand the nature of the electronic interface between bulk-Si and monolayer TMD. As shown in Fig. 2 (b) and (c), various Si-TMD contact regions are simulated with optimized unit cell of the $\mathrm{Si}-\mathrm{MoS}_{2}$ of M-S interface regions for $\mathrm{n} / \mathrm{p}$ - channel operation and contact resistance reduction in the coming discussion. Unit cell optimizations gives the relaxed structure with minimum energy in $\mathrm{Si}-\mathrm{MoS}_{2} \mathrm{M}-\mathrm{S}$ contacts, and a realistic physical separation distance between them can be found.

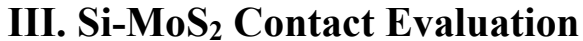

\section{A. Fermi Level Tuning by Doping Si Contact}

Doping Si contact with TMD materials to adjust the channel chemical potential has been demonstrated in our previous experimental work [7]. Fig. 3(a)(b)(c) shows the band structure change of monolayer $\mathrm{MoS}_{2}$ in TMD FET with different type dosage (n-doped:1E21, un-doped, and pdoped:1E21). From the band structure simulation result, the extrinsic dosed type of bulk $\mathrm{Si}$ can effectively adjust the $\mathrm{MoS}_{2}$ Fermi level to close the conduction band by n-type Si contact or the valence band by p-type Si contact. Here, we didn't consider the interface diffusion or surface dipole formation, the band gap keeps the same value $\left(\mathrm{E}_{\mathrm{g}}=\sim 1.8 \mathrm{eV}\right)$ in n- or p-type Si contact condition. The configuration of $\mathrm{MoS}_{2}$ and Si (Fig. 3(d)) are nearly intrinsic. As connected to $\mathrm{Si}$, the $\mathrm{E}_{\mathrm{F}}$ of the $\mathrm{Si}-\mathrm{MoS}_{2}$ contact lies close to the conductance band of $\mathrm{MoS}_{2}$, indicating that $\mathrm{MoS}_{2}-\mathrm{Si}$ is $\mathrm{n}$ typed contact $\left(\Phi_{\mathrm{N}}=0.6 \mathrm{eV}, \Phi_{\mathrm{P}}=1.2 \mathrm{eV}\right)$. Work function shifts versus different type dosage are shown in Fig. 3(e). Noted that P: 1E20 makes original N-contact reversed to P-type one. The higher concentrations of $1 \mathrm{E} 21$ makes $\Phi_{\mathrm{P}}$ drops to $0.2 \mathrm{eV}$ from $0.85 \mathrm{eV}$. Similarly shift effect can also be observed in opposite direction of N-contact. From schematic picture of the DOS of Si-MoS 2 in Fig. 3(f). Compared to un-doped scheme (middle inset), P: 1E21 makes whole DOS profile shifted to higher level, making valence hole state dramatically increase (lower inset), and a $\mathrm{P}+\mathrm{S} / \mathrm{D}$ contact is constructed. In contrast, N-doping yields profile moving downwards to lower energy (upper inset), forming N+S/D contact. Fermi level tuning is also indirectly reflected on the threshold voltage change in current simulation. Compared to un-doped sampling in Fig. 4(a), dosage of 1E-19 shift the threshold voltage from $0.9 \mathrm{~V}$ to a lower position of $0.2 \mathrm{~V}$ with $\mathrm{ON} / \mathrm{OFF}$ ratios exceeding $>10^{4}$ at source-drain voltage $\mathrm{V}_{\mathrm{SD}}=0.1 \mathrm{~V}$.

\section{B. Rc Lowering by Gradient $\operatorname{MoS}_{\mathbf{x}}$ Junction}

Hydrogen treatment on TMD materials to compact $\mathrm{Si}$ $\mathrm{MoS}_{2}$ interface has been reported in our previous experimental work [7]. Fig. 5. shows the schematic of simulated Si-MoS $\mathrm{S}_{2}$ structure with dichalcogenide vacancy by Hydrogen treatments, where the replacement rate of Sulfur by hydrogen was marked with SV0, SV1, SV2 $(25 \%, 50 \%$, $100 \%$ ). Fig. 6. (a) shows the average electron density along $\mathrm{z}$ projected on the $\mathrm{x}-\mathrm{y}$ plane of $\mathrm{Si}-\mathrm{MoS}_{2}$ contacts with and without dichalcogenide vacancy treatments. With SV2 treatment, the nearest physical distance between the Si atoms and the chalcogenide atoms is highly reduced to $1.46 \AA$ and the tunneling barrier $\Phi_{\mathrm{TB}}$ decrease from 0.54 Ry to $0.23 \mathrm{Ry}$, as displayed in Fig. 6. (b). In fact the lower $\Phi_{\text {ТВ }}$ is associated with covalent bond formation. Fig. 7 shows the bond population at interface of SV2 compared to other metalic$\mathrm{MoS}_{2}$. Obviously dichalcogenide vacancy changes interface bonding type from ionic bond of 0.39 in Si-S to covalent bond of value 0.52 in Mo-Si, close to that in inner Mo-S and in Ti-S with 0.52 and 0.59 . Table 2 benchmarks the sequence of normal industry metal contacts with $\mathrm{MoS}_{2}$. Si-monolayer $\mathrm{MoS}_{2}$ with dichalcogenide vacancy takes advantage of comparable tunneling barrier height and electron densities with metalic-TMD contacts. Fig. 8. list the calculated Schottky barrier height of Si monolayer $\mathrm{MoS}_{2}$ under different dichalcogenide vacancy conditions. The dichalcogenide vacancy metalizes the contact interfacial surface, lowering the chemical potential. Hence the original n-type Si-contact was transferred into a P-type one. Note that as replacement rate $\sim 60 \%$, an intrinsic-type junction takes place, indicating a bipolar transistor can be fabricated in the future. Fig. 9. compares the simulated contact resistance of un- and with SV1- Si- monolayer $\mathrm{MoS}_{2}$. The curve clearly display the lowering tunneling barrier induced by dichalcogenide vacancy. In this case, the resistance drops from $75.3 \Omega-\mu \mathrm{m}$ to $55.8 \Omega-\mu \mathrm{m}$ (gain $33 \%$ ) with $50 \%$ dichalcogenide vacancy process, close to the reduction of $29 \%$ in $\Phi_{\text {Тв. Fig. } 10 \text { and }}$ Fig. 11 shows the output $I_{D}-V_{G}$ and $I_{D}-V_{D}$ curve for dichalcogenide vacancy engineer on monolayer $\mathrm{MoS}_{2}$ FET. Both of the forward and reverse - $\mathrm{I}_{\mathrm{ON}}$ current are increased, consistent with previous prediction of $\Phi_{\mathrm{TB}}$ reduction. Moreover, a non-linear slope of Schottky contact can be displayed with $\mathrm{I}_{\mathrm{D}}-\mathrm{V}_{\mathrm{G}}$ and $\mathrm{I}_{\mathrm{D}}-\mathrm{V}_{\mathrm{D}}$ (Fig. 10, and 11). The dichalcogenide vacancy slightly drops $\Phi_{\mathrm{P}}$ with more hole injection, and it significantly improves pMOS output current, as shown in Fig. 11.

\section{Conclusions}

An atomic-scale numerical study of $\mathrm{Si}-\mathrm{MoS}_{2}$ contacts is proposed by the First Principle simulation for the first time. The $\mathrm{n}$ - or $\mathrm{p}$ - type channel formation by doping Si-TMD contacts and the SBH reduction of gradient $\mathrm{MoS}_{\mathrm{x}}$ junctions can get well performance and contact in $4 \mathrm{~nm}$ channel length of monolayer $\mathrm{MoS}_{2} \mathrm{FET}$. It points to the possibility of low nm node $\mathrm{MoS}_{2}$ FET using Si-TMD contacts. It further shows a way to give monolayer TMD FETs a new feature of SiTMD contacts in conventional Si process for the first time.

\section{Acknowledgement}

This simulation work was performed by the National Nano Device Laboratories facilities and supported by the Ministry of Science and Technology, Taiwan; Applied Materials, inc and NCTU-UCB I-RiCE program. 


\begin{tabular}{|c|c|c|c|c|c|c|}
\hline Metalic & $\mathbf{N i}$ & - & $\overline{\mathrm{Au}}$ & - & $\mathrm{WTe}_{2}$ & $\mathrm{Si}$ \\
\hline channel & graphene & phosphorene & phosphorene & $\mathbf{M o S}_{2}$ & $\mathbf{M o S}_{2}$ & $\mathbf{M o S}_{2}$ \\
\hline $\begin{array}{c}\text { contact } \\
\text { type }\end{array}$ & $\begin{array}{l}\text { Adequate } \\
\text { doping }\end{array}$ & edge contact & - & Mo vacancy & $\begin{array}{c}\text { heterojunctio } \\
\text { n }\end{array}$ & $\begin{array}{c}\text { doping } \mathrm{Si} / \mathrm{S} \\
\text { vacancy }\end{array}$ \\
\hline $\mathbf{R}_{\mathrm{c}}$ & $30 \Omega \mu \mathrm{m}$ & $25 \Omega \mu \mathrm{m}$ & - & - & - & $56 \Omega \mu \mathrm{m}$ \\
\hline $\mathbf{L}_{\mathrm{g}}$ & - & $10.5 \mathrm{~nm}$ & $7 \mathrm{~nm}$ & $6.3 \mathrm{~nm}$ & $3 \mathrm{~nm}$ & $4 \mathrm{~nm}$ \\
\hline transistor & n-type & n/p-type & n-type & TFET & TFET & n/p-type \\
\hline reference & IEDM 2015 [12.7] & IEDM 2015 [12.1] & IEDM 2015 [12.2] & IEDM 2015 [12.4] & IEDM 2015 [12.3] & this work \\
\hline
\end{tabular}

Table 1. The simulated metalic-semiconductor contacts performance comparison table of novel 2D semiconductor.

(a)

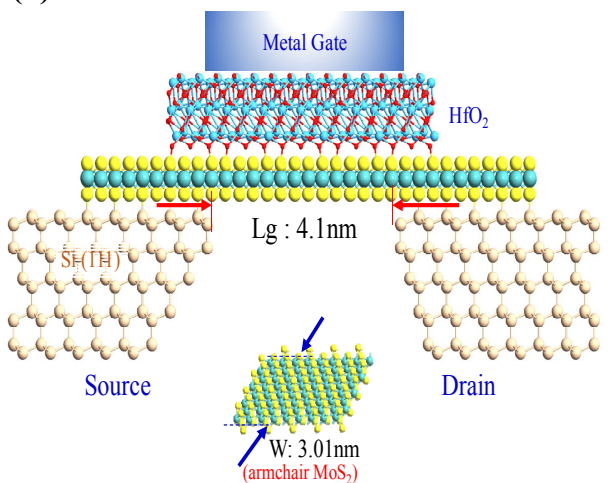

Fig. 2. (a) Simulated structures of sub-5 nm channel length monolayer $\mathrm{MoS}_{2}$ FET with

(b) edge/ (c) side contacts (d) optimized unit cell of the $\mathrm{Si}-\mathrm{MoS}_{2}$ interface regions

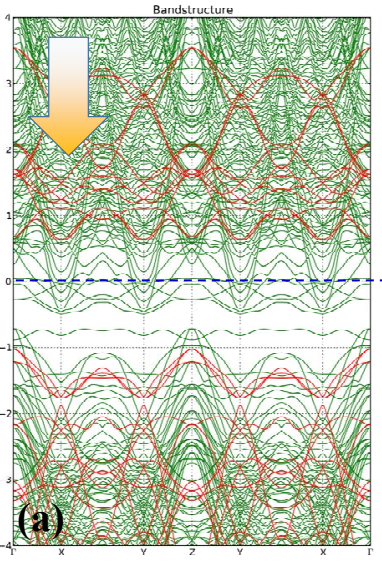

N-doped: Concentration=1E21

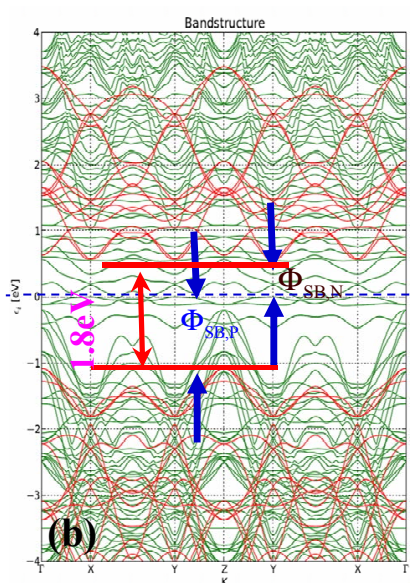

Intrinsic Si-MoS ${ }_{2}$ Contatcs

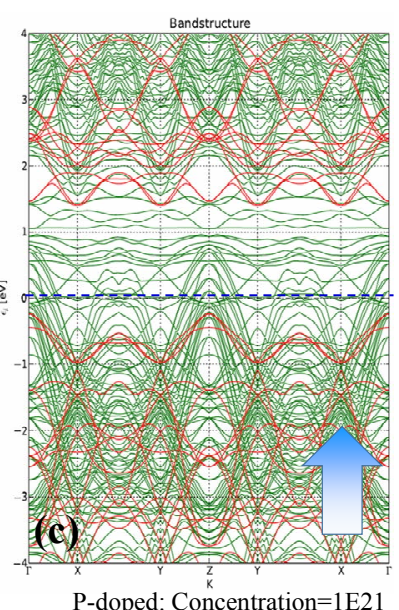

P-doped: Concentration=1E2 1 (d) Unit cell

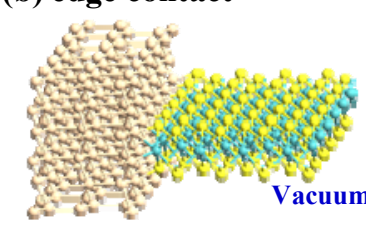

(c) side contact

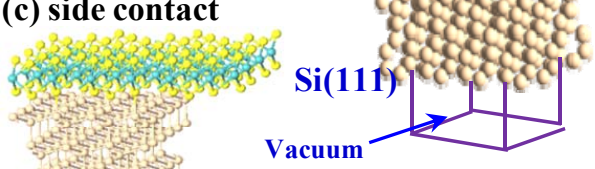

Optimized Geometry

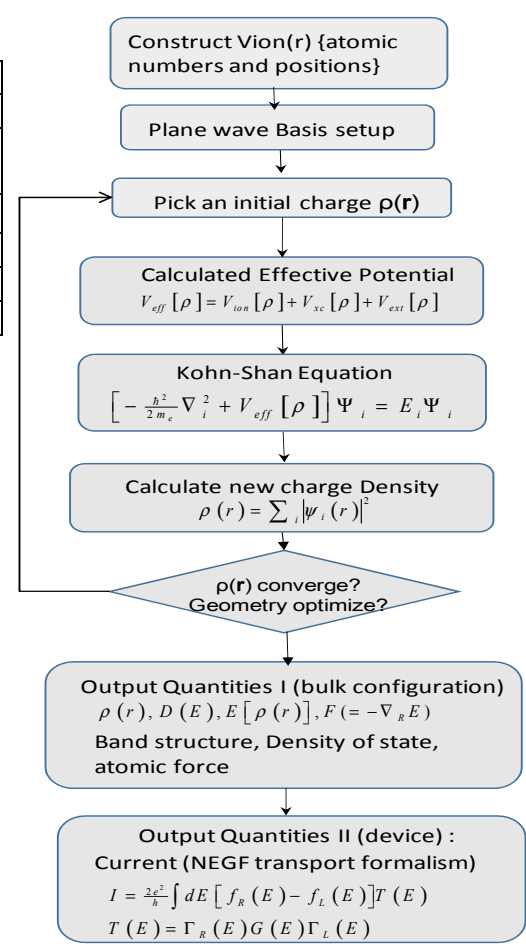

Fig. 1. A Flow chart of simulating sub-5 $\mathrm{nm}$ channel length $\mathrm{MoS}_{2}$ FET.

Fig. 3. (a)(b)(c) Band structure of the metalic- $\mathrm{MoS}_{2}$ interface with different type dosage in Si side contacts. (d) Bulk reference (e) Schottky barrier change vs. different doping condition, $\Phi_{\mathrm{SB}, \mathrm{N} / \mathrm{P}}$ denotes $\mathrm{n} / \mathrm{p}$ - barrier height for electrons/holes.

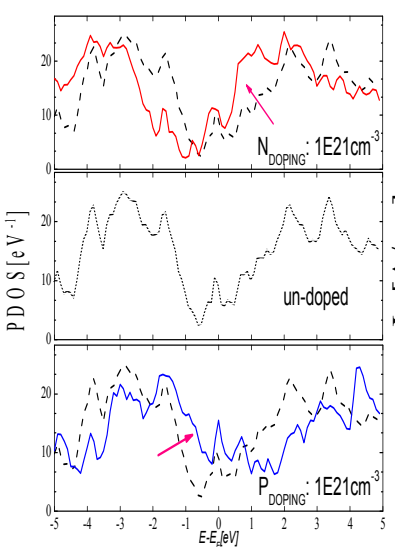

Fig. 3. (f) DOS spectra for $\mathrm{n} / \mathrm{p}$ doping.
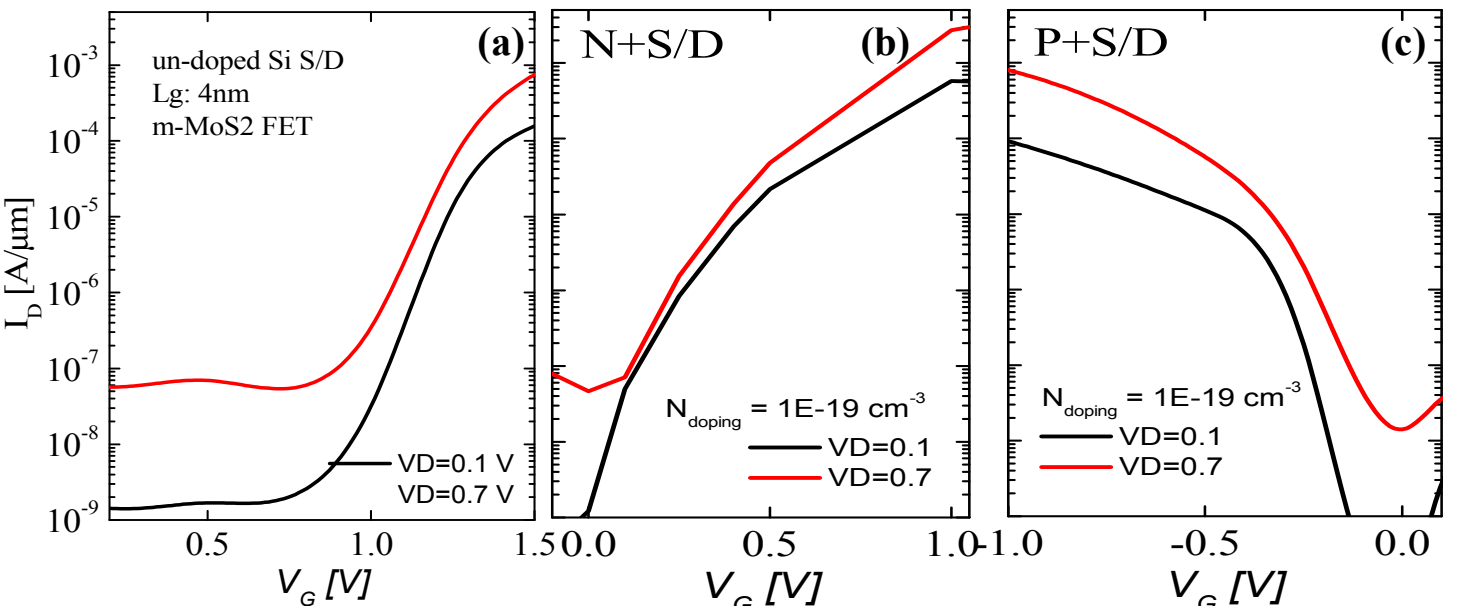

Fig. 4. The simulated $\mathrm{I}_{\mathrm{D}}-\mathrm{V}_{\mathrm{G}}$ of $4 \mathrm{~nm}$ channel length monolayer $\mathrm{MoS}_{2}$ FET with (a) undoped (b) p-doping (c) n-doping Si contacts

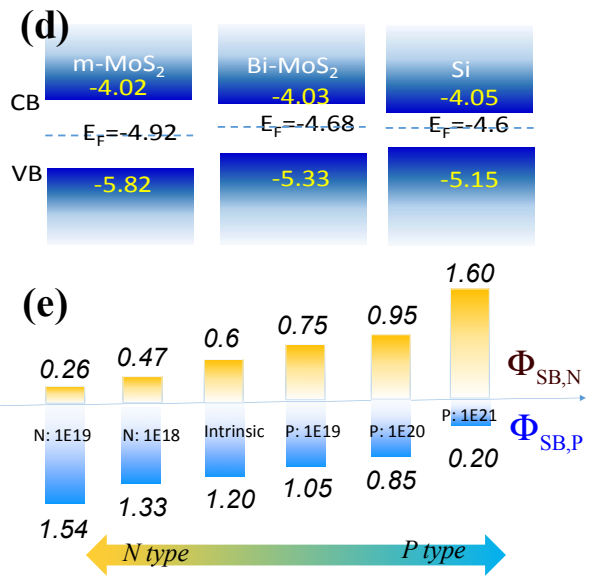




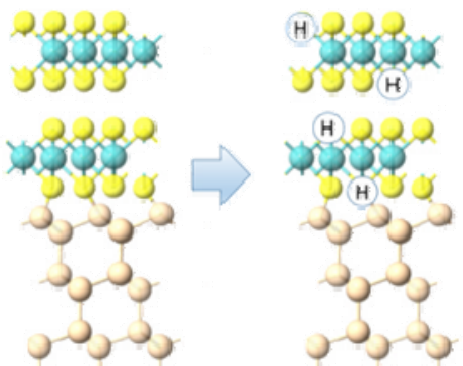

Fig. 5. Schematic of simulated Si-MoS structure with S-vacancy.

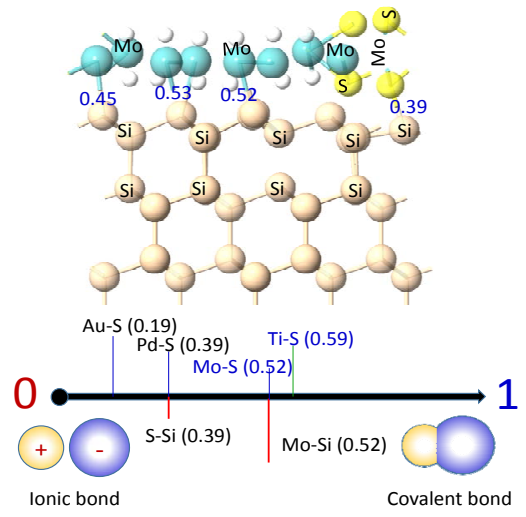

Fig. 7. The covalent bond population at metallic-channel interface.

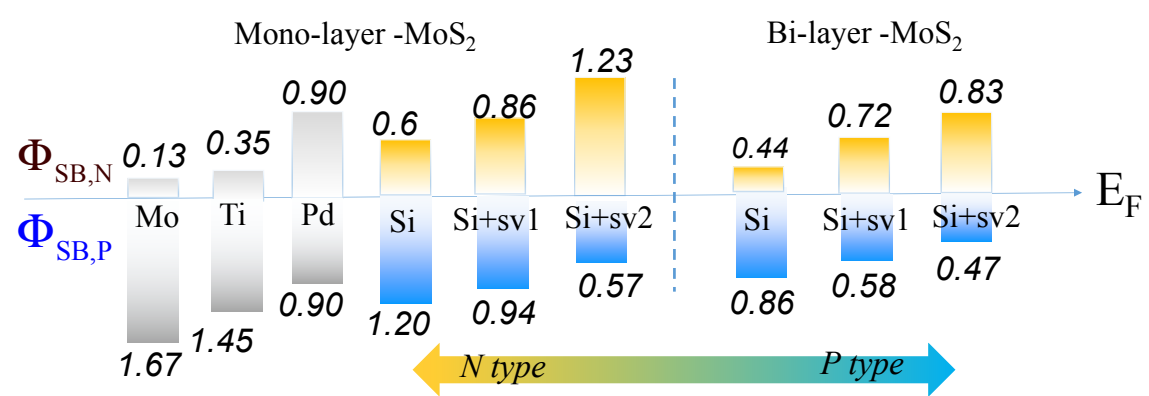

Fig. 8. The calculated Schottky barrier height of Si-monolayer $\mathrm{MoS}_{2}$ under different S-vacancy treatments and other metallic- $\mathrm{MoS}_{2}$.

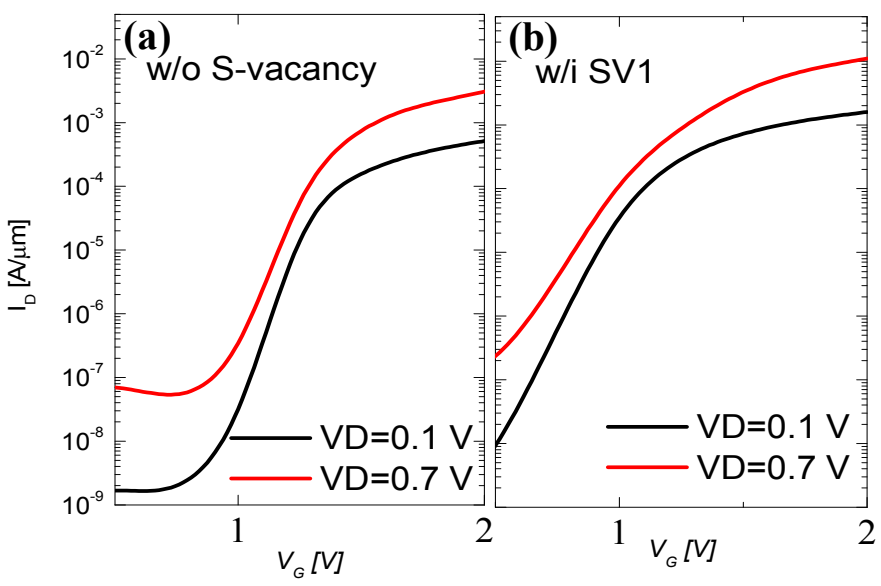

Fig. 10. The simulated $I_{D}-V_{G}$ of $4 \mathrm{~nm}$ channel length monolayer $\mathrm{MoS}_{2}$ FET wi and w/o S-vacancy.

\section{References}

[1] M.C. Chen, et. al., in IEDM Tech. Dig., pp. 831-834, 2015.

[2] B. Radisavljevic, et. al., in Nature Nanotech. vol. 6, pp. 147-150, 2011

[3] B. Radisavljevic, et. al., in Nature Nanotech. vol. 6, pp. 147-150, 2011.

[4] Aron Szabo, et. al., in IEDM Tech. Dig., pp. 297-300, 2015. (12.1)

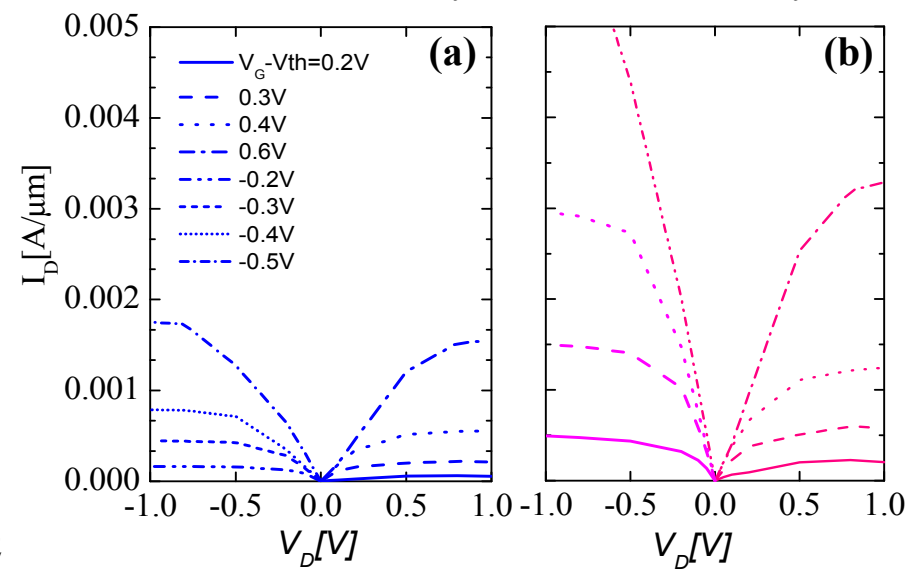

Fig. 11. The simulated $I_{D}-V_{D}$ of $4 \mathrm{~nm}$ channel length monolayer $\mathrm{MoS}_{2}$ FET w/o and w/i S-vacancy.

[5] Teresa Cusati, et. al.,in IEDM Tech. Dig., pp. 321-324, 2015. (12.7)

[6] L. Yang, et. al., in VLSI Symp. Tech. Dig., pp. 238-239, 2014.

[7] K.-S. Li, et. al., in VLSI Symp. Tech. Dig., pp. 52-53, 2016.

[8] A. Nourbakhsh, et. al., in VLSI Symp. Tech. Dig., pp. 54-55, 2016.

[9] Jiahao Kang, et. al., in Phys. Rev. X 4, 031005. 